\title{
Microsymposia
}

MS.10.1

Acta Cryst. (2011) A67, C40

\section{Crystals, Cures and Conundrums}

Alastair J. Florence, ${ }^{\mathrm{a}}$ Norman Shankland ${ }^{\mathrm{a}}$ and Kenneth Shankland, ${ }^{\mathrm{b}} \quad{ }^{a}$ Strathclyde Institute of Pharmacy and Biomedical Sciences, University of Strathclyde, Glasgow, G4 ONR, (U.K.) ${ }^{b}$ Reading School of Pharmacy, University of Reading, PO Box 226, Whiteknights, Reading, RG6 6AP, (U.K.). E-mail: alastair.florence@, strath.ac.uk

X-ray powder diffraction (XRPD) is a powerful tool for the investigation of polycrystalline pharmaceutical solids, being widely applied to phase-identification ("fingerprinting") of solid forms during experimental crystallisation searches during preclinical development screening [1], [2]. With the availability of modern diffractometers, yielding high quality data, plus free and commercial software tools, structure determination from powder diffraction data (SDPD) using global optmisation approaches, XRPD has also become widely used for structural analysis in the absence of suitable single crystal samples. As such, it is increasingly an important element of studies into novel molecular materials [3], polymorphs [4] and solvates [5]. Signifcantly, the structures of many pharmaceutical solids are complex comprising large, flexible molecules often with multiple fragments (co-crystals, salts, solvates), $Z^{\prime}>1$ and possibly disorder. These features contribute to the overall complexity of the problem in terms of both the molecular (internal Degrees of Freedom, DoF) and crystallographic (external DoF) complexity. In order to maximise the chances of successfully locating the global minimum, particulary for such complex problems, a number of strategies can be adopted. Here we will focus, through the use of examples, on steps in the process that contribute to the successful, routine application of SDPD to challenging systems. This will include: data collection approaches; accurate input model generation; implementation of global optimisation calculations and the use of modal torsion angle constraints from the CSD [6]; robust Rietveld analaysis and methods for final structure verification.

[1] G. Barr, W. Dong, C.J. Gilmore, J. Appl. Cryst. 2004. 37, 658-664. [2] A.J. Florence, et al., J. Pharm. Sci. 2003, 92(9), 1930-1938. [3] G.M. Espallargas, et al., J. Am. Chem. Soc. 2007, 129(50), 15606-15614. [4] P. Fernandes, et al., Acta Crystallogr. 2007, E63, O202-O204. [5] P. Fernandes, et al., J. Pharm. Sci. 2007, 96(5), 1192-1202. [6] F.H. Allen, Acta Crystallogr. 2002, B58, 380-388.

Keywords: powder diffraction data, pharmaceuticals, global optimization

\section{MS.10.2}

Acta Cryst. (2011) A67, C40

Solving Structures from Powder Data Using Maximum Entropy and Charge Flipping

Chris Gilmore, ${ }^{a}$ Kenneth Shankland, ${ }^{\mathrm{b}}{ }^{a}$ School of Chemistry, University of Glasgow, Glasgow G12 8QQ, Scotland, (UK). ${ }^{b}$ School of Pharmacy, University of Reading, Reading RG6 6AH, (UK). E-mail: chris.gilmore@glasgow.ac.uk

The solution of crystal structures from powder diffraction data has been revolutionized by the use of model building coupled with global optimization methods and simulated annealing [1]. The charge flipping technique for solving crystal structures [2] has also had a considerable impact in areas such as incommensurate structures, and situations where the space group is uncertain or unknown, and this method is also applicable to powder diffraction data. It is widely presumed that complete data to atomic resolution are needed for success with this technique, but with the incorporation of the maximum entropy formalism [3], the method can be shown to work with data at $c a .2 \AA$ resolution. This combined approach works as follows:

1. Reflection intensities are extracted from the powder diffraction data using either the Pawley or the LeBail method.

2. The reflection data are passed to the Superflip computer program [4] which carries out charge flipping using the powder diffraction options. A total of 100 phase sets, starting from random phase choices, are generated and passed to the maximum entropy program $M I C E$ [5]. In general the solutions generated by Superflip with this data do not converge even after 10000 cycles and/or they have large figures of merit, and cannot be distinguished from each other.

3. Each phase set is subjected to constrained entropy maximization and an associated log-likelihood (LLG) figure of merit is.calculated

4. Electron density maps (in the form of centroid maps [6]) are computed for the top 24 solutions as ranked by LLG.

5. These maps are filtered by histogram matching [7], and the top 5-15 retained and examined.

The method has been used successfully on a number of powder XRD datasets collected from structures of varying molecular and crystallographic complexity of pharmaceutical importance [8]. In some favourable cases, maximum entropy alone (without the use of Superflip) can be successful. The solutions may well prove useful in defining approximate molecular envelopes that can be used as a starting point for model building methods.

The methodology also works for electron diffraction data including two-dimensional data sets.

[1] A.J. Florence, N. Shankland, K. Shankland, W.I.F. David, E. Pidcock, X. Xu, A. Johnston, A.R. Kennedy, P.J. Cox, J.S.O. Evans, G. Steele, S.D. Cosgrove, C.S. Frampton. J. Appl. Cryst. 2005, 38, 249-259. [2] G. Oszlányi, A. Süto. Acta Cryst. 2008, A64, 123-134. [3] C.J. Gilmore, W. Dong, D.L. Dorset. Acta Cryst. 2008, A64, 284-294. [4] L. Palatinus, G. Chapuis J. Appl. Cryst. 2007, 40, 786790. [5] C.J. Gilmore, G. Bricogne, Methods in Enzymology 1997, 277, 65-78. [6] C.J. Gilmore, G. Bricogne, C. Bannister, Acta Cryst. 1990, A46, 297-308. [7] C.J. Gilmore, W. Dong, D.L. Dorset, Acta Cryst. 2005, A64, 284-294 [8] See: http:/www.powderdata.info/data.htm

Keywords: powder diffraction, maximum entropy, charge flipping

\section{MS.10.3}

Acta Cryst. (2011) A67, C40-C41

\section{Macromolecular Powder Diffraction: From Structure to drug} Irene Margiolaki, ${ }^{\mathrm{a}, \mathrm{b}}$ Jonathan Wright, $^{\mathrm{b}}$ Andrew N. Fitch, ${ }^{\mathrm{b}}{ }^{a}$ Department of Biology, Section of Genetics, Cell Biology and Development, University of Patras, GR-26500, Patras, (Greece). ${ }^{\mathrm{b}}$ European Synchrotron Radiation Facility, ESRF, BP220, 38043, Grenoble Cedex, (France). E-mail: imargiola@upatras.gr, margiolaki@esrf.fr

In recent years, modern powder diffraction techniques have been applied to several microcrystalline proteins. The use of high-resolution synchrotron data, together with new analysis procedures, has stimulated exciting progress which is showing that powders can offer unique opportunities for the structural characterization of proteins and are complementary to existing methods. The developments in experimental methods and instrumentation have been absolutely essential, and they will be discussed [1], [2], [3]. Perhaps the most enticing goal for any crystallographer is to solve new structures, and thus we will discuss the progress and prospects for powder diffraction data in this area. We will describe some of the structural refinements which have been carried out for powders using single or multiple pattern fitting and the Rietveld method [1]. The application of the powder diffraction method to phase identification in mixtures and crystal screening [4], [5] will also be illustrated. 